\title{
Status of the detector design studies for $\mathrm{ESS} \nu \mathrm{SB}$
}

\author{
J. Park*, J. Cederkäll, P. Christiansen \\ Department of Physics, Lund University, Box 118, SE-22100 Lund, Sweden \\ E-mail: joochun.park@nuclear.lu.se \\ G. Barrand \\ Laboratoire de l'Accélérateur Linéaire, Université Paris-Sud, CNRS-IN2P3, Orsay, \\ France
}

\section{Bogomilov, G. Petkov, R. Tsenov}

Department of Atomic Physics, St. Kliment Ohridski University of Sofia, Sofia 1504, Bulgaria

\section{T. Ekelöf}

Department of Physics and Astronomy, Uppsala University, Box 516, SE-75105

Uppsala, Sweden

\section{G. Fanourakis, O. Zormpa}

NCSR Demokritos, Box 60037, 15341 Agia Paraskevi, Greece

\section{G. Gökbulut, A. Kayiș Topaksu, M. Oğlakçi}

Faculty of Arts and Science, Physics Department, Cukurova University, 01330 Adana, Turkey

\section{B. Kliček, K. Krhač, M. Stipčević}

Center of Excellence for Advanced Materials and Sensing Devices, Ruđer Bošković Institute, HR-10000 Zagreb, Croatia

The European Spallation Source Neutrino Super Beam (ESS $\nu$ SB) project aims at a discovery of leptonic $\mathrm{CP}$ violation with a precise measurement of the $\mathrm{CP}$ phase angle. $\mathrm{ESS} \nu \mathrm{SB}$ is characterized by an intense neutrino beam to be produced at ESS by a 5-MW proton beam, and the placement of the far detector at the second oscillation maximum. The aims of the near detector for $\mathrm{ESS} \nu \mathrm{SB}$ are neutrino flux and interaction cross section measurements. For this purpose, designs consisting of a fine-grained tracker and a 1-kiloton water Cherenkov detector are under investigation. On the other hand, the far detector will be a water Cherenkov detector with an estimated fiducial volume of 500 kilotons. The design considerations include an evaluation of the stability of the detector hall and excavation sites in deep underground mines. All of the detector simulations are based on frameworks which involve Geant4. A versatile event display toolkit for visualization and physics outreach activities has been developed.

The 21st international workshop on neutrinos from accelerators (NuFact2019)

August 26 - August 31, 2019

Daegu, Korea

${ }^{*}$ Speaker. 


\section{Introduction}

The European Spallation Source Neutrino Super Beam (ESS $\nu$ SB) Project [1] is a future accelerator-based neutrino oscillation experiment design for $\mathrm{CP}$ violation discoveries in the lepton sector. The main driver of $\mathrm{ESS} \nu \mathrm{SB}$ is the ESS facility and its 5-MW proton linear accelerator in Lund, Sweden, which was designed to generate the world's most intense source of pulsed neutrons. In order to meet the physics goals of $\operatorname{ESS} \nu \mathrm{SB}$, several technical upgrades involving the proton beam energy, charge stripping and beam accumulation schemes are necessary.

The now-established neutrino mixing angle $\theta_{13} \sim 10^{\circ}$ for the muon-electron neutrino appearance channel and associated with measuring the Dirac CP violation phase $\delta_{C P}$ has revealed that measuring $\delta_{C P}$ is advantageous near the second oscillation maximum compared to the first. These findings were taken into account at the preliminary conceptual designs of $\mathrm{ESS} \nu \mathrm{SB}$. A dedicated working group for $\mathrm{ESS} \nu \mathrm{SB}$ detector designs aims to reduce systematic uncertainties in signal/background neutrino flux to below $5 \%$, such that the $5 \sigma$ discovery range of $\delta_{C P}$ may increase.

\section{Detector simulation and performance tests}

The ESS $\nu$ SB detector working group has developed EsbRoot for detector simulations and event reconstruction based on the FairRoot framework, an open-source software for simulation, reconstruction and data analysis at the Facility for Antiproton and Ion Research (FAIR). However, for the design of water Cherenkov (WC) detectors envisioned as a near detector (ND) as well as the far detector (FD), an additional open-source simulation package called WCSim, is being used. WCSim is based on Geant4 [2] and ROOT.

A generalised particle source for basic diagnostic tests of the detector geometry and reconstruction algorithms has been used, as well as a Monte-Carlo neutrino event generator GENIE [3] on the estimated neutrino flux from ESS for more realistic event simulations and detector response evaluations. Its outputs have been successfully used as inputs for EsbRoot and WCSim, and the integration of GENIE into EsbRoot is complete. A characterisation of all scattering reaction channels of the neutrinos in active detector materials will be attempted. A prospect of constraining the neutrino flux from ESS with measurements of neutrino-electron reaction channels is being explored.

\subsection{The near detector (ND)}

The purpose of the ND for $\mathrm{ESS} \nu \mathrm{SB}$ is the characterization of the neutrino flux near the production site, which is necessary for reducing the systematic uncertainty of the neutrino flux at the FD. Measuring neutrino interaction cross sections at the aforementioned energy range is also of physics interest.

A proposal for a small-scale WC detector with as the main component of ND was motivated by opportunities to reduce or eliminate systematic uncertainties arising from utilising different detectors and reconstruction algorithms between the ND and the FD. Due to the low neutrino energies, the topologies of neutrino events in the sensitive detector 
volumes are expected to be relatively simple. This phenomenon is beneficial for smallscale NDs whose resolving power of distinguishing multiple sources of radiation is limited due to the finite volumes for particle trajectories and Cherenkov light cone propagation. The presumably low interaction cross section is compensated by the high neutrino beam intensity near the production site. An algorithm for reconstruction of Cherenkov rings in WC detectors of the Super/Hyper-Kamiokande (SK, HK) geometries has already been developed, namely fiTQun [4]. This software performs a maximum-likelihood method using the charge and time information from photomultiplier tube (PMT) hits on the walls of the WC detector. A dedicated scheme for tuning the reconstruction parameters in fiTQun to a ND geometry for $\mathrm{ESS} \nu \mathrm{SB}$ is being established.

In order to supplement particle identification and momentum reconstruction of the ND, a design based on the Super Fine Grained Detector (SFGD) [5] is under investigation. This detector consists of many $1 \mathrm{~cm}^{3}$ plastic scintillator cubes with wavelength-shifting fibers in three orthogonal projections for 3D position information (see Fig. 1a). A dipole magnetic field to surround the SFGD volume is also envisioned. Reconstruction of SFGD events requires both the sequential hit pattern recognition and trajectory reconstruction. The pattern recognition is achieved by the graph traversal method, and an open-source track fitting algorithm toolkit GENFIT [6] has been employed (see Fig. 1b and 1c). It was found that GENFIT requires tuning and additional modifications to improve the reconstruction accuracy of low-energy events for $\mathrm{ESS} \nu \mathrm{SB}$.

A supplementary ND subsystem in the form of a nuclear emulsion detector is being explored. An emulsion cloud chamber with water as the target material may be deployed to measure neutrino-water cross sections, as being pursued by the NINJA collaboration [7].

a.

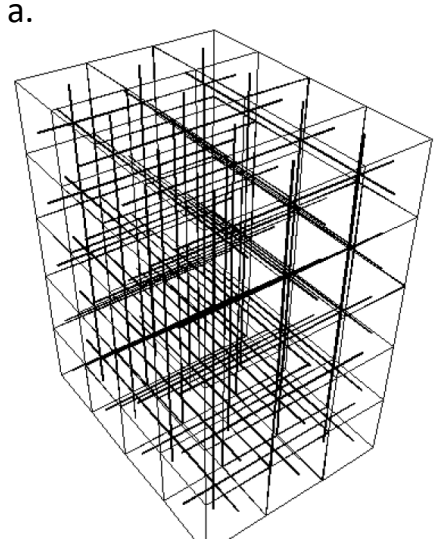

b.

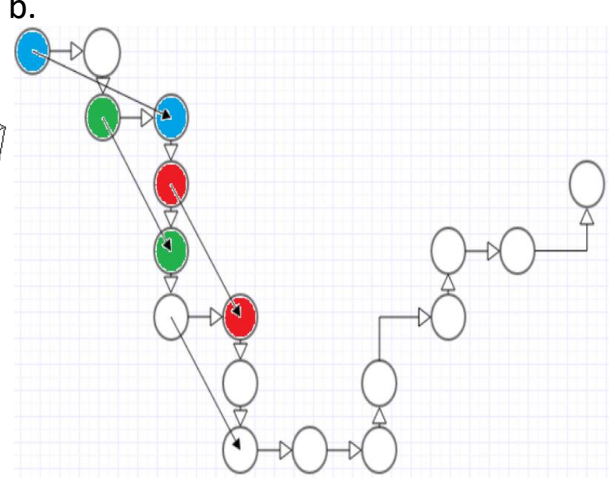

c.

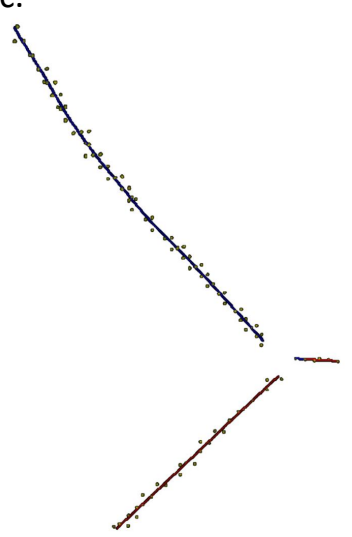

Figure 1: a. Sub-volume of SFGD simulated in EsbRoot. The wavelength-shifting fibers inside the plastic scintillator cubes are visible. b. Schematic diagram of the hit pattern recognition with graph traversal. c. Simulated SFGD hit pattern and fitted tracks using GENFIT for a muon and two protons from a $600-\mathrm{MeV}$ neutrino event, in an external magnetic field of $0.5 \mathrm{~T}$. 


\subsection{The far detector (FD)}

The FD for ESS $\nu \mathrm{SB}$ is a large water Cherenkov detector, based on the MEgaton Mass PHYSics (MEMPHYS) design [8]. Studies on the expected performance of the FD were conducted in the same way as WC-ND, via EsbRoot, WCSim and fiTQun. Certain reconstruction and analysis strategies such as fiducial volume and timing cuts are expected to be transferrable, and it remains to be seen whether the strategies for tuning reconstruction algorithms can be applied in a consistent manner between the WC-ND and the FD.

On the other hand, the dimensions and the location of the FD depend on the conditions of the deep underground sites and additional factors which may be out of the physics scope. As part of the Underground Neutrino Research Infrastructure (UNRI) project, two active mines in Garpenberg and Zinkgruvan are considered for the ESS $\nu$ SB FD. Both sites are located in Sweden. Geological surveys of potential sites for stability after boring large volumes of rock to accommodate massive detectors are compulsory. For active mines, care must be taken to eliminate mutual disturbance in the rock volumes due to mining operations, which lead to stability issues. A feasibility study of the Zinkgruvan mine was conducted, and the preliminary conclusion was that the rock mass was competent enough for further examination. Further recommendations and risk assessments are underway.

\section{Event visualization}

An effort to develop an informative and versatile event display software for ESS $\nu$ SB was undertaken in parallel with the detector design studies. Such a tool is also valuable for education and outreach activities for a long-term scientific project such as ESS $\nu$ SB. As a product of this aim, EsbRootView is capable of rendering 3D graphics of nearand far-detector hits and geometries, and is compatible with multiple operating systems: Linux/X11, Windows/Win32, macOS/Cocoa, iOS and Android. EsbRootView is built by using the "softinex" library developed at Orsay [9]. Two event display examples from EsbRootView are shown in Fig. 2.

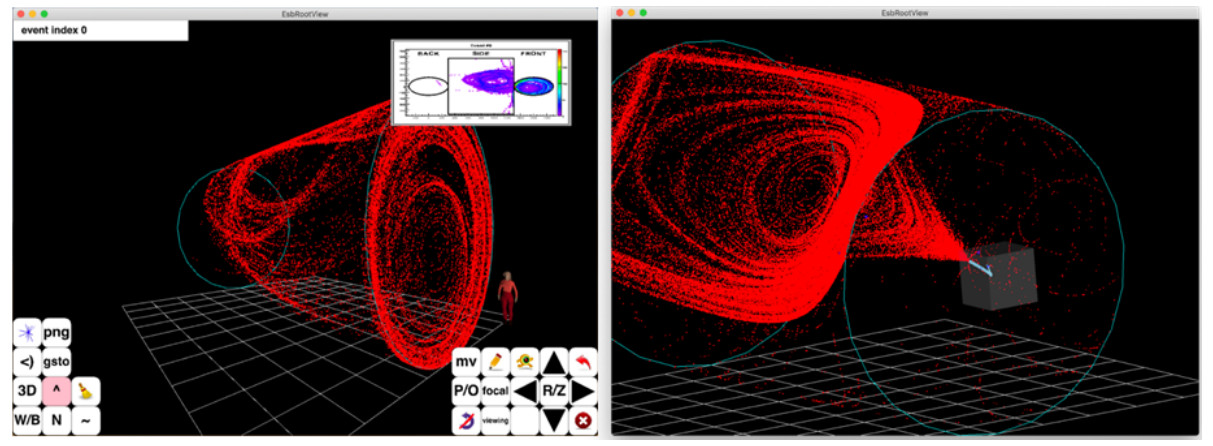

Figure 2: Event displays from EsbRootView on near-detector geometries. Left: WC detector with PMTs illuminated (red dots) from a muon and its decay. Many display and customization features have been implemented. Right: propagation of a muon neutrino event inside the SFGD volume (grey cube) to the adjacent WC near detector volume. 
EsbRootView is also capable of replaying an event from simulated detector hits as a video clip with adjustable speed and time range settings, based on the simulation geometry and time information. By accessing the truth information, there is an additional feature to distinguish different sources of radiation and be able to apply selection cuts in order to isolate them. A graphical user interface, initial startup scripts and command-line prompts are being implemented. Publication of EsbRootView on online application stores for smartphones and tablets is a possibility, pending a discussion within the collaboration.

\section{Summary and outlook}

The working group for the detector performance of $\operatorname{ESS} \nu \mathrm{SB}$ has been simulating and evaluating options for both ND and FD. Multiple simulation, analysis and software frameworks were adopted from various external collaborations in order to expedite the design process, but will eventually be integrated into the final software framework EsbRoot. A powerful and informative event display toolkit for detector diagnostics and public outreach purposes is available on multiple platforms as EsbRootView.

The expected performance of a small-scale WC detector and SFGD are being examined as a function of the geometry. The priority is on tuning the reconstruction software to yield the highest attainable accuracy on lepton identification and best correlated migration matrices, in the context of low-energy neutrino events from ESS. The location of the FD site and the final dimensions of the FD are being discussed with civil and mining engineering considerations.

\section{Acknowledgments}

This project is supported by the COST Action EuroNuNet "Combining forces for a novel European facility for neutrino-antineutrino symmetry-violation discovery". It has also received funding from the European Union's Horizon 2020 research and innovation programme under grant agreement No 777419, and the Bulgarian National Science Fund grant No. DCOST01/8.

\section{References}

[1] E. Baussan et al., Nucl. Phys. B 885, 127 (2014).

[2] S. Agostinelli et al., Nucl. Instr. Meth. Phys. Res. A 506, 250 (2003).

[3] C. Andreopoulos et al., Nucl. Instr. Meth. Phys. Res. A 614, 87 (2010).

[4] A. D. Missert (T2K Collaboration), Jour. Phys. Conf. Ser. 888, 012066 (2017).

[5] A. Blondel et al., Jour. Instrum. 13, P02006 (2018).

[6] C. Höppner et al., Nucl. Instr. Meth. Phys. Res. A 620, 518 (2010).

[7] T. Fukuda et al., Prog. Theo. Exp. Phys. 2017, 063 C02 (2017).

[8] L. Agostino et al., Phys. Rev. ST Accel. Beams 16, 061001 (2013).

[9] G. Barrand, J. Phys. Conf. Ser. 513, 022002 (2014). 